\title{
Epigenetic Changes Of Oncogenic And Anti-Oncogenic Factors Encoding Gene During Gynaecological Tumour Progression
}

\author{
Takuma Hayashi $^{1,2}$, Norihiko Kikuchi ${ }^{1}$, Miki Kawano ${ }^{2,3}$, Tomoyuki Ichimura ${ }^{4}$, \\ Dorit Zharhary ${ }^{5}$, Yae Kanai ${ }^{6}$, Nobuo Yaegashi ${ }^{7}$, \\ Tanri Shiozawa ${ }^{1}$, Ikuo Konishi ${ }^{8}$ \\ '(Dept. of Obstetrics and Gynaecology, Shinshu University School of Medicine, Nagano, Japan) \\ ${ }^{2}$ (Dept. of Medical Technology, International University of Health and Welfare, Chiba, Japan) \\ ${ }_{3}^{3}$ (Dept. of Health Science, Kyushu University Graduate School of Medicine, Fukuoka, Japan) \\ ${ }^{4}$ (Dept. of Obstetrics and Gynaecology, Osaka City University Graduate School of Medicine, Osaka, Japan) \\ ${ }^{5}$ (SIGMA-Aldrich Israel, Rehovot, Israel) \\ ${ }^{6}$ (Pathology Division, Keio University School of Medicine, Tokyo, Japan) \\ ${ }^{7}$ (Dept. of Obstetrics and Gynaecology, Tohoku University Graduate School of Medicine, Miyagi, Japan) \\ ${ }^{8}$ (National Hospital Organization Kyoto Medical Centre, Kyoto, Japan)
}

\begin{abstract}
Hypoxia is known to play key roles in the development and progression of tumours. We previously demonstrated that breast cancer type 1 susceptibility protein (BRCA1) and S100 calcium-binding protein A4 (S100A4), critical molecules for tumour initiation and metastasis, were differential expressed in ovarian cancer. Therefore, we examined the mechanisms of the BRCA1 and S100A4 expression in ovarian carcinoma cells, with particular attention paid to the effects of hypoxia. The expression levels of BRCA1 and S100A4 were found to correlate with the invasiveness of ovarian carcinoma cells in vitro and in vivo, and the expression of BRCAI and S100A4 gene was associated with epigenetic changes in the regulatory regions of BRCAI and S100A4 cording genes in ovarian carcinoma cell and tissues. The expression of S100A4 was increased under hypoxia, and then was associated with elevated invasiveness. In addition, exposure to hypoxia reduced the methylation of hypoxia-response elements (HRE) of the S100A4 gene in a time-dependent fashion, in association with the enhancing binding activity of hypoxia inducible factor-1 $\square$ (HIF-1 $\square \square$ to a methylationfree HRE in ovarian carcinoma cells. These findings indicate that hypoxia-induced hypomethylation plays an essential role in S100A4 over expression and the epigenetic transformation of ovarian carcinoma cells into the "metastatic phenotype".
\end{abstract}

Keywords: S100A4, BRCAl, Hypoxia, hypomethylation, Ovarian carcinoma

\section{Introduction}

Breast cancer type 1 susceptibility protein (BRCA1) and BRCA2 are normally expressed in the cells of breast and other tissue, where they help repair damaged DNA, or destroy cells if DNA cannot be repaired. If BRCA1 or BRCA2 itself is damaged by a BRCA mutation, damaged DNA is not repaired properly, and this increases the risk for breast cancer. S100 calcium-binding protein A4 (S100A4) belongs to the S100 family of calcium binding proteins ${ }^{1-3}$. S100A4 may function in motility, invasion, and tubulin polymerization. The indication that the expression of BRCAl and S100A4 gene in different malignant tumour cells is strongly correlated with tumour progression and aggressive metastatic phenotypes ${ }^{4}$.

Later on, a potent metastasis regulating role of S100A4 was demonstrated convincingly by use of various approaches and animal models in different research facilities ${ }^{1,3}$. More recent data associates the upregulation of S100A4 both in ovarian tumour, and stroma cells with poor prognosis and survival of patients with different types of cancer ${ }^{5-9}$. Various immune cells, macrophages, neutrophils, certain types of lymphocytes, dendritic and mast cells and human endothelial cells express and release S100A4 into the extracellular space ${ }^{10}$. Specifically, we focus on the mammalian methylation and demethylation activities in the ovarian carcinoma progression, and the problem of transmitting epigenetic information across tumour cell divisions and generations. In this report, we will discuss the emerging importance of the epigenetic alterations of BRCAl and S100A4 genes in the management of gynaecological tumours, ovarian cancer.

\section{The Epigenetic Changes Of Anti-Oncogenic Factor BRCA1 Gene In Gynaecological Tumours}

The epigenetic changes regulate gene expression. Although the physiological significance of DNA methylation in the transcriptional silencing of anti-oncogenic factor cording gene is recognized, genome-wide hypomethylation has also been reported in human malignancy and has been associated with genetic instability 
11,12. The epigenetic changes of tumor suppressor gene, BRCA1 and hypomethylation of metastasis associated gene, S100A1 are focused to clarify biological characteristics of ovarian cancer. BRCAl is anti-oncogenic factor, which plays a crucial role in the repair of DNA damages and its abnormality is responsible for hereditary ovarian cancer syndrome ${ }^{13-16}$. To clarify possible involvement of BRCRl in the development of sporadic ovarian neoplasms, we analyzed the BRCAl expression, loss of heterozygosity (LOH) and it's promoter methylation in normal ovarian surface epithelium and 119 epithelial ovarian tumours ${ }^{17}$. The lowered expression of BRCAl was normally observed in carcinoma, the differential expression of BRCA1, which correlated protein along with epigenetic changes, might play a key role in development of sporadic ovarian carcinomas ${ }^{17}$. From prognostic analysis, patients with ovarian carcinoma negative for BRCAl expression showed favorable prognosis. If anything, the cisplatin sensitivity and apoptosis would be observed among patients with ovarian carcinoma negative for BRCAl expression ${ }^{17}$. According expression of BRCAl might be an important biomarker for cisplatin resistance in ovarian carcinoma.

\section{The Epigenetic Changes Of Metastasis Associated Factor S100A4 Gene In Gynaecological Tumours}

The S100A4 protein, which belongs to calcium binding S100 protein family, has reportedly been associated with cell motility and invasion ${ }^{3,4}$. The hypoxia reportedly attenuates the "metastatic phenotypes" of ovarian carcinoma cells and found the enhancing expression of S100A4 under hypoxia ${ }^{18-20}$. We investigated expression of S100A4 and subcellular localization in 113 epithelial ovarian neoplasms and analyzed its prognostic significance in patients with ovarian carcinoma. Pathological analysis showed that both cytoplasmic and nuclear expressions of S100A4 were significantly stronger in carcinomas than those in benign and borderline tumours. Patients with ovarian carcinoma, which markedly expressed nuclear S100A4, showed a significantly shorter survival than lowered expression of nuclear S100A4. Moreover, the in vivo analysis demonstrated that the nuclear expression of S100A4 is involved in the aggressive behavior of ovarian cancer cells. Bisulfite sequence experiment demonstrated that hypomethylation of S100A4 encording gene was associated with over expression of S100A4 ${ }^{21,22}$. Since hypoxia increased ovarian cancer invasiveness with enhance S100A4 expression, we examined the change of methylation status of S100A4 under hypoxia. Hypoxia increases hypomethylation of $1^{\text {st }}$ intron of S100A4 gene and increased bindings between hypoxia inducible factor-1 $\square$ (HIF-1 $\square \square$ and hypomethylated hypoxia response elements (HREs) in $1^{\text {st }}$ intron of S100A4 gene ${ }^{21,22}$. On the other hand, in case of myometrium, certain mesenchymal tumour cells that express caveolins, which are a family of integral membrane proteins, have been shown to be more aggressive and metastatic, because of a potential for anchorage-independent growth. From our findings up-regulation of S100A4 and/or integral membrane proteins expression might be associated with hypomethylation, along with increased the malignancy during the gynaecological tumour progression.

\section{Discussion}

Expression level of BRCA1 is also relevant to clinical treatment against ovarian cancer. Patients with lowered BRCA1 expressing sporadic ovarian cancer, had longer median survival times under the treatment with platinum drugs in comparison with higher BRCA1 expression. Our previous study showed that the nuclear expression of S100A4 was an independent prognostic factor in patients with ovarian cancer ${ }^{21,22}$. Further report showed that the expression of nuclear S100A4 in combination with nuclear HIF-1 $\alpha$ is a potential biomarker of poor prognosis. Accordingly, the presence of hypoxic conditions might upregulate S100A4 expression, producing an unfavorable prognosis. The translocation of S100A4 between the cytoplasm and the nucleus has been reported in human normal, mesenchymal tumour and malignant tumour cells ${ }^{23,24}$. The expression of nuclear S100A4 has been reported to be implicated in the regulation of gene transcription either through direct DNA binding or its interaction with other DNA-binding proteins ${ }^{23,24,25}$. The upregulation of S100A4 expression was associated with hypomethylation, along with increased malignancy during gynaecological tumours; ovarian cancer and mesenchymal tumour progression ${ }^{26}$. Research findings suggest that the nuclear expression of S100A4 combined with HIF-1 $\alpha$ may be a significant biomarker and could be a molecular target for clinical treatment against ovarian cancer.

The significant activity of poly (ADP-ribose) polymerase (PARP) inhibitors in the treatment of germline $B R C A$ epigenetic character-associated ovarian carcinoma, which represents $\sim 15 \%$ of high-grade serous cases, has recently led to European Medicines Agency and food and drug administration approval of olaparib. Further research addressing the optimal material for BRCA and S100A4 epigenetic analyses in gynaecological tumours; ovarian cancer, uterine mesenchymal tumours is needed.

\section{Conclusion}

Recent research report showed that the upregulation of S100A4 expression was associated with hypomethylation, along with increased malignancy during gynaecological tumours; especially ovarian cancer 
progression. We also found that exposure to hypoxia increased the hypomethylation of the first intron of S100A4 and increased the binding of HIF- $1 \alpha$ in gynaecological tumours. These results indicate that hypoxiainduced hypomethylation plays an important role in gene overexpression during gynaecological tumour progression.

\section{Acknowledgements}

This work was supported in part by Grants-in-Aid for Scientific Research to T.H. (No. 24592510) from the Ministry of Education, Science and Culture of Japan.

\section{References}

[1]. N. Ambartsumian, M. Grigorian, and E. Lukanidin, Genetically modified mouse models to study the role of metastasis-promoting S100A4(mts1) protein in metastatic mammary cancer. Journal Dairy Research, 72, 2005, 27-33.

[2]. S.C. Garrett, K.M. Varney, D.J. Weber, and A.R. Bresnick, S100A4, a mediator of metastasis. Journal Biological Chemistry, 281, 2006, 677-680.

[3]. D.M. Helfman, E.J. Kim, E. Lukanidin, and M. Grigorian, The metastasis associated protein S100A4: role in tumour progression and metastasis. British Journal Cancer, 92, 2005, 1955-1958.

[4]. A. Ebralidze, E. Tulchinsky, M. Grigorian, A. Afonayeva, V. Senin, E. Revazova, and E. Lukanidin, Isolation and characterization of a gene specifically expressed in different metastatic cells and whose deduced gene product has a high degree of homology to a Ca2+-binding protein family. Genes and Development, 3, 1989, 1086-1093.

[5]. P.S. Rudland, A. Platt-Higgins, C. Renshaw, C.R. West, J.H. Winstanley, L. Robertson, and R. Barraclough, Prognostic significance of the metastasis-inducing protein S100A4 (p9Ka) in human breast cancer. Cancer Research, 60, $2000,1595-1603$.

[6]. L. Mazzucchelli, Protein S100A4: too long overlooked by pathologists? American Journal Pathology, 160, $2002,7-13$.

[7]. E.J. Kim, and D.M. Helfman, Characterization of the metastasis-associated protein, S100A4. Roles of calcium binding and dimerization in cellular localization and interaction with myosin. Journal Biological Chemistry, 278, 2003, 30063-30073.

[8]. J.F. Cui, Y.K. Liu, B.S. Pan, H.Y. Song, Y. Zhang, R.X. Sun, J. Chen, J.T. Feng, Z.Y. Tang, Y.L. Yu, H.L. Shen, and P.Y. Yang, Differential proteomic analysis of human hepatocellular carcinoma cell line metastasis-associated proteins. Journal Cancer Research Clinical Oncology, 130, 2004, 615-622.

[9]. E. Missiaglia, E. Blaveri, B. Terris, Y.H. Wang, E. Costello, J.P. Neoptolemos, T. Crnogorac-Jurcevic, and N.R. Lemoine, Analysis of gene expression in cancer cell lines identifies candidate markers for pancreatic tumorigenesis and metastasis. International Journal Cancer, 112, 2004, 100-112.

[10]. T. Cabezón, J.E. Celis, I. Skibshøj, J. Klingelhöfer, M. Grigorian, P. Gromov, F. Rank, J.H. Myklebust, G.M. Mælandsmo, E. Lukanidin, and N. Ambartsumian, Expression of S100A4 by a variety of cell types present in the tumor microenvironment of human breast cancer. International Journal Cancer, 121, 2007, 1433-1444.

[11]. A.P. Feinberg, and B. Vogelstein, Hypomethylation distinguishes genes of some human cancers from their normal counterparts. Nature, 301, 1983, 89-92.

[12]. Y. Miki, J. Swensen, D. Shattuck-Eidens, P.A. Futreal, K. Harshman, S. Tavtigian, Q. Liu, C. Cochran, L.M. Bennett, W. Ding, A strong candidate for the breast and ovarian cancer susceptibility gene BRCA1. Science, 266, 1994, 66-71.

[13]. S.C. Rubin, I. Benjamin, K. Behbakht, H. Takahashi, M.A. Morgan, V.A. LiVolsi, A. Berchuck, M.G. Muto, J.E. Garber, B.L. Weber, H.T. Lynch, J. Boyd, Clinical and pathological features of ovarian cancer in women with germ-line mutations of BRCA1. New England Journal of Medicine, 335, 1996, 1413-1416

[14]. J. Boyd, Y. Sonoda, M.G. Federici, F. Bogomolniy, E. Rhei, D.L. Maresco, P.E. Saigo, L.A. Almadrones, R.R. Barakat, C.L. Brown, D.S. Chi, J.P. Curtin, E.A. Poynor, W.J. Hoskins, Clinicopathologic features of BRCA-linked and sporadic ovarian cancer. JAMA, 283, 2000, 2260-2265.

[15]. Y. Ben David, A. Chetrit, G. Hirsh-Yechezkel, E. Friedman, B.D. Beck, U. Beller, G. Ben-Baruch, A. Fishman, H. Levavi, F. Lubin, J. Menczer, B. Piura, J.P. Struewing, Effect of BRCA mutations on the length of survival in epithelial ovarian tumors. Modan B; National Israeli Study of Ovarian Cancer. Journal Clinical Oncology, 20, 2002, 463-466.

[16]. C. Zhou, J.L. Smith, J. Liu, Role of BRCA1 in cellular resistance to paclitaxel and ionizing radiation in an ovarian cancer cell line carrying a defective BRCA1. Oncogene, 22, 2003, 2396-2404.

[17]. C. Wang, A. Horiuchi, T. Imai, S. Ohira, K. Itoh, T. Nikaido, Y. Katsuyama, I. Konishi, Expression of BRCA1 protein in benign, borderline, and malignant epithelial ovarian neoplasms and its relationship to methylation and allelic loss of the BRCA1 gene. Journal Pathology, 202, 2004, 215-223.

[18]. R. Osada, A. Horiuchi, N. Kikuchi, J. Yoshida, A. Hayashi, M. Ota, Y. Katsuyama, G. Melillo, I. Konishi, Expression of hypoxiainducible factor 1alpha, hypoxia-inducible factor 2alpha, and von Hippel-Lindau protein in epithelial ovarian neoplasms and allelic loss of von Hippel-Lindau gene: nuclear expression of hypoxia-inducible factor 1alpha is an independent prognostic factor in ovarian carcinoma. Human Pathology, 38, 2007, 1310-1320.

[19]. S. Shahrzad, K. Bertrand, K. Minhas, B.L. Coomber, Induction of DNA hypomethylation by tumor hypoxia. Epigenetics, 2, 2007, $119-125$.

[20]. R. Xie, D.S. Loose, G.L. Shipley, S. Xie, R.L. Bassett Jr, R.R. Broaddus, Hypomethylation-induced expression of S100A4 in endometrial carcinoma. Modern Pathology, 20, 2007, 1045-1054.

[21]. N. Kikuchi, A. Horiuchi, R. Osada, T. Imai, C. Wang, X. Chen, I. Konishi, Nuclear expression of S100A4 is associated with aggressive behavior of epithelial ovarian carcinoma: an important autocrine/paracrine factor in tumor progression. Cancer Science, 97, 2006, 1061-1069.

[22]. A. Horiuchi, T. Hayashi, N. Kikuchi, A. Hayashi, C. Fuseya, T. Shiozawa, I. Konishi, Hypoxia upregulates ovarian cancer invasiveness via the binding of HIF-1 $\square$ to a hypoxiainduced, methylation free hypoxia response element (HRE) of S100A4 gene. International Journal Cancer, 131, 2012, 1755-1767.

[23]. K. Flatmark, K.B. Pedersen, J.M. Nesland, H. Rasmussen, G. Aamodt, S.O. Mikalsen, K. Bjornland, O. Fodstad, G.M. Maelandsmo, Nuclear localization of the metastasis-related protein S100A4 correlates with tumour stage in colorectal cancer. Journal of Pathology, 200, 2003, 589-595.

[24]. M. Saleem, M.H. Kweon, J.J. Johnson, V.M. Adhami, I. Elcheva, N. Khan, B. Bin Hafeez, K.M. Bhat, S. Sarfaraz, S. ReaganShaw, V.S. Spiegelman, V. Setaluri, H. Mukhtar, S100A4 accelerates tumorigenesis and invasion of human prostate cancer through the transcriptional regulation of matrix metalloproteinase 9. Proceeding National Academic Science USA, 103,2006, 14825-14830. 
[25]. M.A. Bookman, C.B. Gilks, E.C. Kohn, K.O. Kaplan, D. Huntsman, C. Aghajanian, M.J. Birrer, J.A. Ledermann, A.M. Oza, K.D. Swenerton. Better therapeutic trials in ovarian cancer. Journal of National Cancer Institute 106(4), 2014, dju029. doi: 10.1093/jnci/dju029.

[26]. G. Zhang, M. Li, J. Jin, Y. Bai, C. Yang, Knockdown of S100A4 decreases tumorigenesis and metastasis in osteosarcoma cells by repression of matrix metalloproteinase-9. Asian Pacific Journal Cancer Prevention. 12(8), 2011, 2075-2080. 